\title{
AGENT-BASED LOAD BALANCING FOR MOBILE ROBOT APPLICATIONS
}

\author{
Ralf Kluthe, Wolfgang Obelöer, Claus Grewe \\ Medical University of Lübeck, D-23538 Lübeck
}

\section{Introduction}

There is an increasing demand for mobile autonomous robots, e.g. in the field of service applications. For the use of mobile service robots in real environments, complex and reactive sensing-perception-action systems are necessary. Many of these models were already developed and/or are subject of current research (Mertsching, et al., 1997), (Wasson, et al., 1998). On the other hand, questions on how these models can be integrated into the software architectures for mobile robots as well as the management of system resources necessary in mobile systems are not well studied. Especially the latter requires mechanisms to handle the different timeliness and priorities of the normally parallel tasks even in dynamic environments.

Especially for algorithms based on high resolution sensors, high computational power is needed which enforces the usage of parallelisms. In addition, typical applications in this area show a dynamic behavior due to changes in the environment. Therefore, load balancing has to be a basic part of such a system.

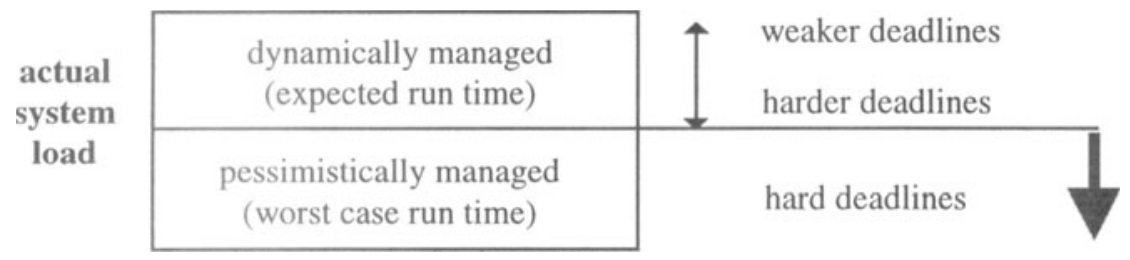

Figure 1. Task Assignment and Load Management Approach.

Due to the dynamic load characteristics of mobile robot applications, it is not well suited to assign and schedule all tasks in a way that the maximum resource requests are handled (worst case approach). The disadvantage of this approach is that system resources may be wasted and that sometimes the maximum resource requests are hard to determine (Bonasso, et al., 1998). In order to reduce the pessimistically managed algorithms, a suitable load management is required. The aim of this management is to reduce the portion of pessimistically managed algorithms (that are algorithms with hard deadlines) to a minimum and handle the rest dynamically (see figure 1). 
Therefore, a suitable software architecture is proposed, where the load balancing of mobile robot applications (section 2) is done by mobile agents (section 3 ). This paper shows a mechanism for migrating processing entities with real-time constraints, which is used by the load balancing approach. For the realization, an existing multiagent environment for load balancing of parallel applications is extended so that it can be used for mobile robot applications (section 4). Finally, first measurements are presented (section 5).

\section{Mobile Autonomous Robots}

Software systems for autonomous robots are normally decomposed into several layers. Here, the Three-Layer Architecture is a widely applied model (Hasemann, 1996). The three abstraction layers are deliberation, sequencing, and reactive behavior (figure 2).

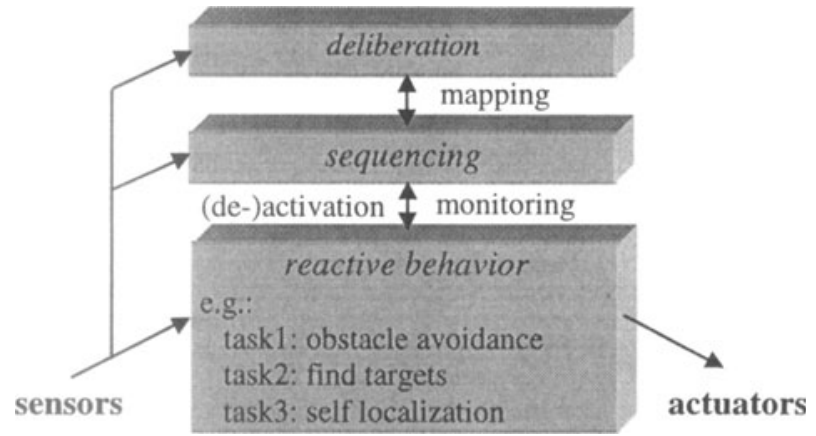

Figure 2. Three-Layer Architecture for Robot Control.

The major tasks of the deliberative layer are planning, scheduling, and resource handling. Activities of this layer correspond to long term planning as well as plan adaptations. The sequencing layer represents tactics, called task nets, i.e. pre-defined, partially ordered sets of actions. The sequencing layer selects appropriate tactics and starts the associated reactive behaviors according to the relationships within the task nets. The reactive behavior layer executes the behavior tasks, activated by the sequencing layer. Several robots have been programmed using some variations of this architecture, e.g. Saphira, 3T, ATLANTIS, LAAS (Bonasso, et al., 1998). In the reactive behavior layer, algorithms stay in close contact with the environment. On this level, complex control involves reflexive and reactive responses, such as object avoidance or object tracking, characterized by high frequency closed loop interaction with the environment. Hence, algorithms used here underlay real-time constraints.

In most software architectures used today, the algorithms respectively the behavior tasks are scheduled in a simple round-robin manner, thus a fixed cycle rate is used. But normally, the behavior tasks have different temporal orders depending on the robot state. So, it is reasonable that at high robot velocity the cycle rate of an obstacle recognition algorithm is higher than that for an object recognition algorithm for person tracking. Hence, modern robot software architectures have to support multirate computation (Wasson, et al., 1998). Furthermore, for a reasonable use of system 
resources, like CPU utilization, cycle rates may be dynamically increased or decreased, e.g. the cycle rate of an obstacle recognition algorithm is proportional to the velocity of the robot.

Due to the increasing demand on high-resolution sensors like laser ranger or cameras, algorithms become more complex. In addition, they are computationally expensive and characterized by a number of computation paths. In this area data flow graphs are normally used for modeling. Often the algorithms are biologically motivated as e.g. attention or fovea control in human perception systems. So, the processing of the data flow chains depends on the dynamics of the environment (Mertsching, et al., 1997) and the robot state. Due to these facts, data flow chains are activated or terminated dynamically. For example, if a special obstacle recognition is needed or some extracted features in the sensor data refer to obstacles, a data flow for further processing is activated additionally. After the obstacle is passed the data flow can be terminated.

Summarized, from system point of view, the reactive part is characterized by the following points:

- Dynamic changes in the environment and attention-based control-mechanisms within the algorithms cause dynamic loads.

- Algorithms can be modeled using data flow graphs and have to fulfil real-time constraints.

- Due to different temporal orders of the algorithms, multi-rate computation occurs.

- Algorithms, especially those for high resolution sensors, are computationally expensive and therefore powerful hardware platforms are required.

Thus, software architectures for mobile robots have to offer mechanisms that allow a suitable exploitation of the system resources. Especially mobile systems have to handle limited resources like computation power and battery energy. Therefore, special management mechanisms like load balancing without violating real-time constraints are unavoidable.

\section{Hierarchical Agent Based Approach}

Here a hierarchical agent based approach for handling dynamic load by migration is presented. Therefore, a load model is defined and a hardware platform is proposed.

\subsection{Load Model and Hardware Platform for Mobile Robot Software}

Robot application software is normally decomposed into several tasks as shown in figure 2. Depending on the current context, the sequencing and planning units, for simplification called robot control units, map the tasks to the hardware and control it. In order to describe the data and control dependencies of one task, a data flow paradigm is used. Thus, the computation model can be considered as a system that dynamically maps data flow chains to the system hardware. Dynamic load can be caused by outer and inner reasons. That means, currently mapped data flow chains can be manipulated in several ways (outer reasons). For example, data flow chains can be started and stopped or the accuracy of the modeled algorithms can be increased or 
decreased. Inner reasons are changes of load by the behavior task, e.g. robot velocity increases and as a consequence the cycle rate of an obstacle recognition algorithm.

Furthermore, the approach has to support suitable hardware platforms. The characteristics of used algorithms ranges from simple, computation intensive operations like convolution in vision systems to complex operations used in searching, matching, or planning algorithms. For complex operations, general-purpose processors are appropriate, whereas for computation intensive operations special hardware, e.g. DSP systems, or general multi-processor systems are used. Here embedded cluster systems consisting of off-the-shelf components (e.g. PCs) connected via a fast network offer an attractive solution. These cluster systems have a good price/performance ratio and special hardware is easy to integrate. Thus, for the presented approach these hardware platforms will be assumed.

\subsection{Multiagent Environment}

As mentioned, an online load balancing approach is presented that handles the resulting imbalances of system load by migration. This implies that migration of data flow nodes is done in a way that real-time constraints are not affected. This can be handled by separating data flow node assignment and scheduling (Krishna, 1997). That means, data flow node assignment and migration is done without real-time constraints. To schedule the allocated data flows, scheduling algorithms offered by the real-time operating system are used. Thus, data flow nodes can be migrated to processing nodes which have enough computation power to fulfil the changed requirements of a data flow. But migration can also be used for compaction, leading to processors that can be switched off (idle mode). For example, a Pentium II 450 MHz has a power consumption of about 30 watts with full load and 2.4 watt in idle mode ( 0.8 watt in deep sleep mode) (Intel, 1998). Thus, battery power can be saved or a new mapping can be done more easily.

Here, mobile agents are a promising solution to solve the load balancing problem respectively the migration problem. The mobility allows an agent to "transport" a data flow node to another processing node. In addition, the mobility can be used to source out tasks, e.g. "long-term" planning tasks, to remote host systems. Due to their autonomy, agents may perform a suitable action by themselves, like reducing the accuracy of their operation. But they may also cooperate with other agents to find a solution like finding a target node for a migration. In addition, agents are pro-active, and they may perform additional tasks like an administrative service in order to measure computation power consumed by a data flow node.

To handle the data flows by agents, a hierarchical multiagent environment is proposed (figure 3 ). The environment allows to model reactive behavior of mobile robots and it offers services like obstacle avoidance to the robot control layer. These services are modeled by data flows. The robot control acquires services with certain constraints, e.g. obstacle avoidance with a maximum cycle rate, by requesting a dispatcher agent. The dispatcher agent has to start the services and to guarantee the requested constraints. After a service is started, the robot control layer manipulates and monitors the functional behavior of the services.

If a service violates its constraints or a service cannot be started due to too few hardware resources, the dispatcher agent has to interact with the robot control to find a 
solution. For example, the robot control could order the dispatcher agent to lower the accuracy or the cycle rate of services. Additionally, the dispatcher agent can be forced to find an own solution. So, the dispatcher agent does an online load balancing in a way that important services (high priorities or hard deadlines) are able to fulfil their constraints. Services with weaker constraints are allowed to violate their deadlines.

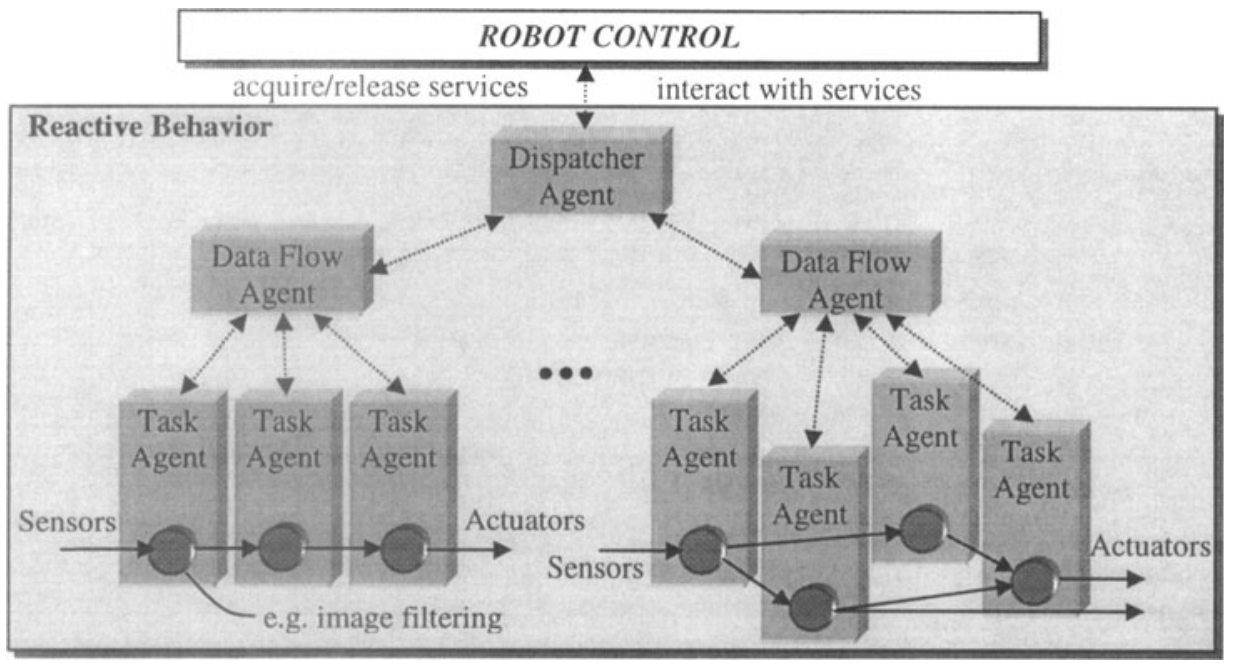

Figure 3. Hierarchical Multiagent Environment.

The dispatcher agent starts a service by ordering a data flow agent. Each data flow agent is responsible for a single service. The data flow agent initiates the mapping of the data flow nodes to the hardware. In addition, it monitors the runtime behavior (deadlines, CPU power consumption, etc.) and interacts with the dispatcher agent if something fails within the service. Data flow agents cooperate among each other to coordinate their actions.

For mapping a service, a data flow agent assigns a task agent to each data flow node. Each task agent is responsible for one data flow node and monitors its runtime behavior. The task agents are mobile and so they can carry or migrate the data flow node to a processing node where the operation is supported.

\section{Realization}

For a realization of the described approach, a multiagent environment with migration and online load balancing capabilities has to be implemented. As a base, the existing multiagent environment FLASH is used. In this section, the environment and the required extensions are described.

\subsection{FLASH}

FLASH (Flexible Agent System for Heterogeneous Cluster) is a framework which offers programmers a platform for the creation of distributed, load-balanced 
applications (Obelöer, et al., 1998) for cluster systems. The user decomposes a problem into several subtasks and assigns each subtask to a mobile FLASH agent. The FLASH agents are responsible for the efficient execution of the given subtasks. Therefore, they travel through a cluster system, e.g. searching for free resources. Without any further information, FLASH uses a simple policy in order to balance the application caused load over the system. Additionally, the user is able to specify alternative load-balancing schemes and/or to involve knowledge about the special application. In general, FLASH supports the range from totally user-transparent to completely user-controlled load balancing and the facility to control single features, e.g. the choice of migration destination.

The FLASH environment consists of four cooperating basic components (see figure 4). On the top of the system one or more system agents observe the whole cluster system and maintain up-to-date information about it. If any relevant changes occur, they inform the local node agents on all participating cluster nodes. Node agents serve as well-known communication partners for the mobile user agents on every visited node. Additionally, the node agents know and maintain all local interfaces to system services, which are the fourth basic component of FLASH. If any user agent needs a special service, e.g. a connection to a high-speed network, it contacts the local node agent for help. This mechanism hides the real, machinespecific implementation of a service and hence supports the heterogeneous approach of FLASH. For example, FLASH cooperates with an external load management system, which is based directly on the underlying operating system and supplies system specific load indices. For this purpose the node agents are residing immobile on the nodes. However, for fulfilling dedicated jobs they can create messenger agents. Other important tasks of node agents are the maintenance of up-to-date information about locally residing user agents and support of centralized decisions.

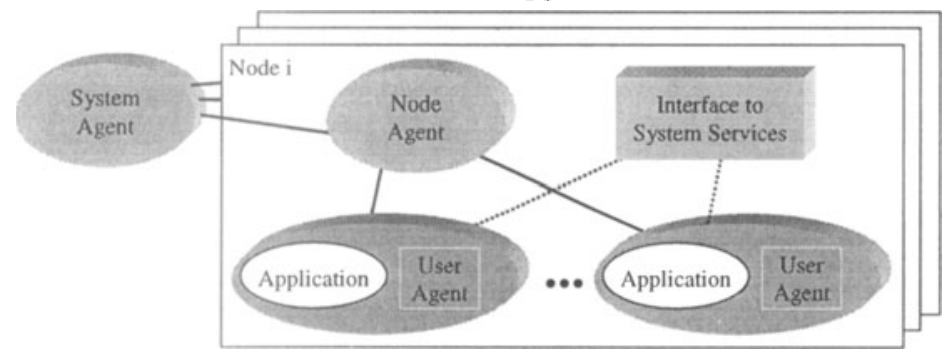

Figure 4. Structure of FLASH.

As shown in figure 4, each user agent contains a single subtask of the application. The agent has to take care for the efficient execution of the subtask. Therefore, it takes into account node capacities, the current load state of the nodes as well as occurring internal and external events, e.g. interactions with other agents or system services. User agents have the possibility to execute the job either directly as a thread in the agent context or to search for a native implementation of the function in the cluster system (accessible via system services). This mechanisms will be explained in the following section. 


\subsection{Realization of FLASH}

Currently, FLASH is mostly written in Java and uses the agent system Voyager (ObjectSpace, 1998) which is completely implemented in Java, too. Voyager offers some unique features (Kiniry, Zimmerman., 1997) and provides the structures and methods for the management and handling of distributed objects. Therefore, it has several kinds of communication types, location transparent handling of remote method invocations, a global name space, and migration facilities. A special Voyager object is the so-called "agent" which is able to cause a migration by itself. On top of this object class the FLASH agents were implemented.

As mentioned, the programmer is able to realize a job in Java or to apply native functions. In FLASH, a native function is accessible via a system service interface. Typically this option is chosen when a node owns special hardware (e.g. a DSP board) or if the computation performance offered by Java is insufficient. Native functions are written in $\mathrm{C}$ or $\mathrm{C}++$ and compiled for a specific machine architecture. Thus, Java applications - and consequently FLASH agents - can overcome performance losses resulting from the interpretation of Java Bytecode by the Java Virtual Machine.

\subsection{Extension of FLASH}

FLASH was originally designed for load balancing of parallel applications without real-time constraints. To use FLASH for mobile robot applications, extensions are required.

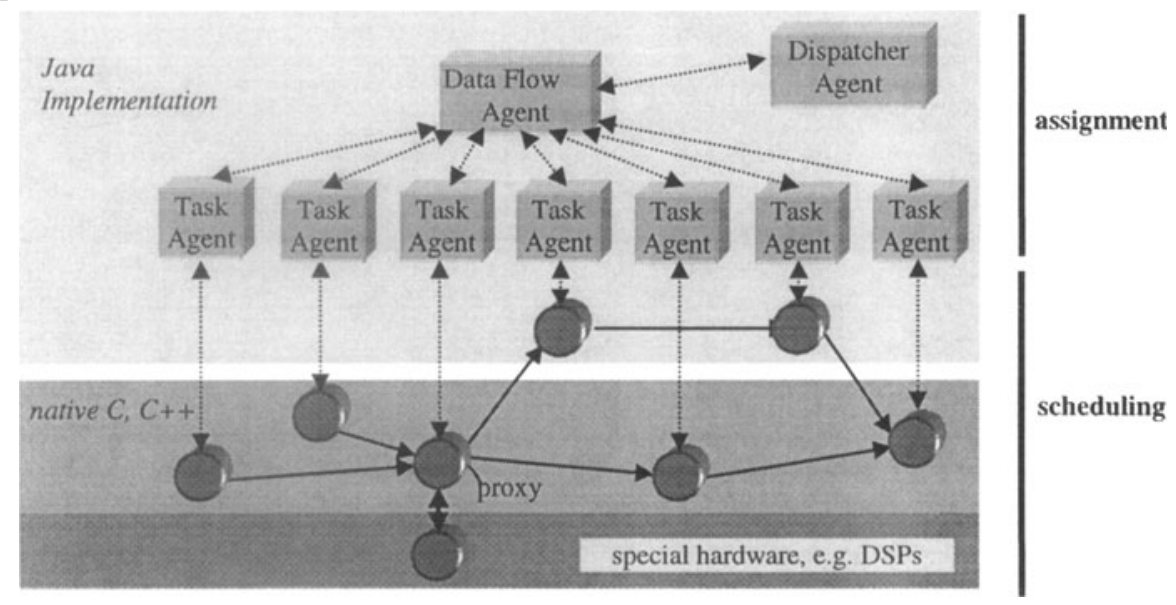

Figure 5. Extension of FLASH.

As mentioned, a separation between data flow node assignment and scheduling is done. Hence, a loosely-coupled mechanism between task agents and data flow nodes is provided (in FLASH, application subtasks are part of the agent, see figure 4). So task agents represent the interface between the agent environment and the real-time algorithms (see figure 5). Real-time scheduling of the data flows is done by the underlying operating system. Thus, agents can run concurrently without directly influencing the data flow. 
Due to performance bottlenecks of Java and the absence of needed algorithms, data flow nodes written in native code have to be supported. A data flow node wrapper is introduced, making it possible to combine different implementations within a data flow. The interaction between Java and native code is provided by the Java Native Interface (JNI). Special hardware is supported by a proxy, whose interface is compatible to that of normal data flow nodes. The proxy handles communication between the data flow and the algorithms mapped to the special hardware.

\section{Measurements}

An efficient realization of the described mobile robot application essentially depends on the appropriate choice of well-suited mechanisms and tools. For this reason, in the first step a distributed emulation environment for the lower $\mathrm{C} / \mathrm{C}++$ parts (figure 5) was realized. The prototype helps to explore the feasibility of migrating data nodes without deadline violations. The environment consists of communicating processes (one per cluster node). All data flow nodes of one processor reside in such a process. These processes have a high priority, i.e. they are almost the exclusive users of CPU resources. This allows to implement alternative scheduling strategies independent from the underlying operating system.

The prototype is able to emulate the temporal behavior of several distributed data flows mapped to a cluster system. Therefore, the emulation schedules the CPU demands of synthetic data flow nodes according to their given priority. In addition, the testbed performs the synthetic load as well as the caused network traffic. Currently, a prioritized round-robin strategy is implemented, but further strategies can be added in a simple manner. For migration a protocol, described in the following section, was implemented. Afterwards, first measurement results are presented.

\subsection{Migration Protocol for the $\mathrm{C} / \mathrm{C}++$ Part}

The implemented migration protocol allows to migrate stateless data nodes without destroying the real-time constraints of the data flows.

First, the data node which has to be migrated is cloned at the destination cluster node. The clone rebuilds all connections to the successors and predecessors in the data flow. Then, all participating data nodes are informed about the clone. For a short time the predecessors send their messages to both, the original and the cloned data node. Both nodes fulfil their job and send results to the same successors. A successor takes the first incoming result and discards the second one. After getting the first valid result from the cloned node, the successors accept results from it exclusively. As soon as all successors accept messages from the cloned node only, the original data node stops sending further results. Then the predecessors are informed, which stop sending messages to the original data node. Afterwards the original node is terminated.

\subsection{First Measurements}

The presented measurements were done on a cluster of dual-processor PCs (Pentium II, $330 \mathrm{MHz}$ ) interconnected via Myrinet (Boden, et al., 1995). 
For exploration of the described migration scheme two data flows (DF1 and DF2) were configured, which are started periodically (see figure 6). DF1 has a higher priority than DF2. During measurements the time slice in the emulation was adjusted to $10 \mathrm{~ms}$. The medial CPU usage of the single cluster nodes was evaluated for the specified CPU demands. The CPU usage of cluster nodes 0 and 2 is in the range of $35 \%$, the usage of cluster node 1 is about $75 \%$.

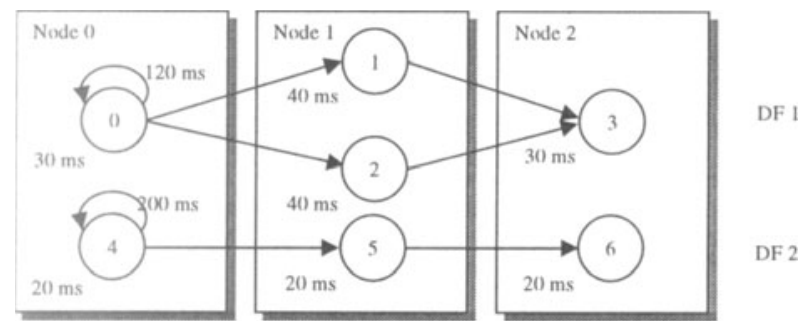

Figure 6. Test Configuration.

For the following measurement it is assumed that the robot aims to increase its velocity. Therefore, it is necessary to reduce the periodicity of DF1 (e.g. to $100 \mathrm{~ms}$ ). These leads to a higher load on all participating cluster nodes. In addition, the second data flow starves because the higher prior data flow demands more and more CPU resources on cluster node 1 . Hence, a migration of a data node is necessary in order to guarantee the functionality of DF2. Here, the migration of data node 1 to cluster node 2 was chosen. Figure 7 shows the CPU usage for time intervals of 0.5 seconds.

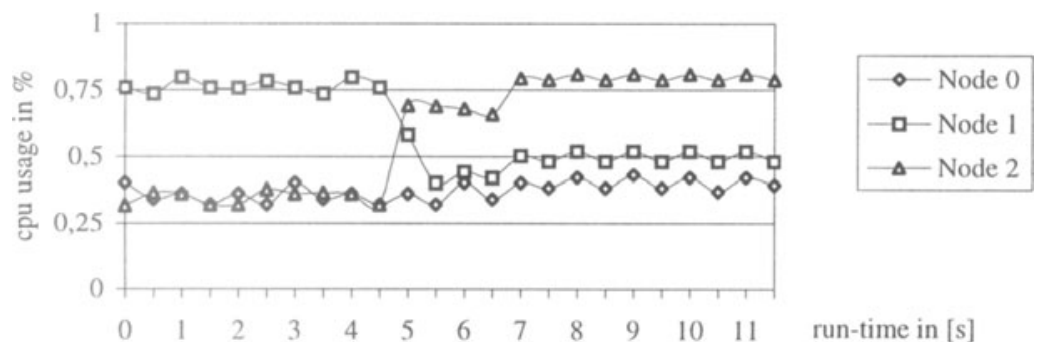

Figure 7. CPU Usage with Migration of a Data Node.

The migration of data node 1 is initiated after 5 seconds and takes $75 \mathrm{~ms}$ protocol overhead (without setup-time for a thread or process). As can be seen in figure 7 the load of cluster node 1 decreases (from ca. $75 \%$ to $40 \%$ ) while that of node 2 increases. After seven seconds (two seconds after the migration order) the periodicity of the source data node 0 is reduced to $100 \mathrm{~ms}$ which causes an increased load in the system. During the migration as well as afterwards the deadline of DF1 was met.

The migration time of the FLASH agent was measured separately. Here, a migration time of averaged $32 \mathrm{~ms}$ was measured for an idle system. The presented measurements are a first confirmation that the proposed agent-based approach is able to fulfil the requirements of real-time applications in the field of service robots. 


\section{Conclusion}

Today high computation power is needed for mobile robot applications, which can only be offered by parallel systems. These applications cause dynamic load fluctuations due to the dynamics of the environment. Hence, a load management has to be applied which considers the special demands of real-time applications.

In this paper a hierarchical multiagent-based management system for robot applications is presented, which consists of a computation and management part. The computation part executes the data flows of the robot application, scheduling is done by the underlying operating system. Here, several realizations of data nodes (e.g. in Java, $\mathrm{C}$, or $\mathrm{C}++$ ) can be combined in a uniform manner. The management part hides implementation details and administrates the arising resource demands.

Currently, the implementation is based on FLASH (Flexible Agent System for Heterogeneous Cluster), a Java-based development platform which was created as a framework for distributed applications. This paper discusses the necessary extensions of FLASH for combining Java-based and native data nodes. In addition, an emulation environment was realized, which serves as a testbed for the evaluation of distributed and cooperative load management strategies for mobile robot applications. Especially, the possibility of migrating a data node from one computation node to an other was explored.

For the future the evaluation of different load management heuristics as well as the use of the approach in a real mobile robot is planned.

\section{References}

Boden, N.J; et al. (1995). "Myrinet: A Gigabit-per-Second Local-Area Network." IEEE Micro 15(1), 29-36.

Bonasso, R.P.; Kortenkamp, D.; Murphy, R. (1998). Artificial Intelligence and Mobile Robots, Menlo Park, California: The MIT Press.

Hasemann, J.-M. (1996). A Control Architecture for Intelligent Robots Based on Graph Manipulation for Planning, Monitoring, Execution, Intention Switching, and Fault Recovery, Dissertation, Espoo: Technical Research Center of Finland.

Intel (1998). "Datasheet Pentium II Processor at $350 \mathrm{MHz}, 400 \mathrm{MHz}$, and $450 \mathrm{MHz}$." Intel Corp., Ordering Number 243657-002, http://www.intel.com.

Kiniry, J.; Zimmerman, D. (1997). “A Hands-On Look at Java Mobile Agents." IEEE Internet Computing 1(4), 21-30.

Krishna, C. M.; Shin, K. G. (1997). Real-Time Systems. New York: McGraw-Hill.

Mertsching, B.; Bollmann, M. (1997). "Visual Attention and Gaze Control for an Active Vision System." in Progress in Connectionist-Based Information Systems (ed. Kasabov N.; Kozma, R.; et al.), Berlin: Springer, 76-79.

Obelöer, W.; Grewe, C.; Pals, H. (1998). "Load Management with Mobile Agents." in Proceedings of the 24th EUROMICRO Conference, Los Alamos: IEEE Computer Society, vol. 2, 1005-1012.

ObjectSpace (1997). ObjectSpace Voyager Core Package. Tech. Overview, ObjectSpace, Inc.

Wasson, G.; Bonasso, R. P.; Kortenkamp, D. (1998). "Integrating Active Perception with an Autonomous Robot Architecture." in Proceedings of the 2nd International Conference on Autonomous Agents, 325-331. 